\title{
Correction to: Aphid-induction of defence-related metabolites in Arabidopsis thaliana is dependent upon density, aphid species and duration of infestation
}

\author{
Simon Hodge $\mathrm{e}^{1,2} \odot \cdot$ Mark Bennett $^{1} \cdot$ John W. Mansfield ${ }^{1} \cdot$ Glen Powell $^{1,3}$
}

Published online: 26 August 2019

(c) Springer Nature B.V. 2019

\section{Correction to: \\ Arthropod-Plant Interactions (2019) 13:387-399 \\ https://doi.org/10.1007/s11829-018-9667-0}

The authors would like to include the following changes in the published article.

The authors concede that errors were made in the nomenclature of the indole glucosinolates that make interpretation of results problematic.

The four generally-accepted indole glucosinolates in $A$. thaliana are: indol-3-ylmethylglucosinolate, 1-methoxyindol-3-ylmethylglucosinolate, 4-hydroxyindol-3-ylmethylglucosinolate and 4-methoxyindol-3-ylmethylglucosinolate (Brown et al. 2003). These four compounds were detected in our study, and not the mistyped 3-methoxy derivative, and the glucosinolate we abbreviated as 3MIND is actually the parent indole glucosinolate, indol-3-ylmethylglucosinolate. Another more minor issue was that the methylsulphinylalkyl glucosinolates and methylthioalkyl glucosinolates should have a bracket in their names to indicate that it is the entire group that is attached to position 4. Finally, all the glucosinolates should strictly have "glucosinolate" as the final part of their name. Therefore, Table 1 of the original manuscript required modification and should read as given below.

The original article can be found online at https://doi.org/10.1007/ s11829-018-9667-0.

Simon Hodge

simon.hodge68@gmail.com

1 Division of Biology, Imperial College London, South Kensington, London SW7 2AZ, UK

2 Present Address: Future Farming Centre, Lincoln University, Canterbury, New Zealand

3 Present Address: NIAB EMR, New Road, East Malling, Kent, UK
The authors also accept that the codes they have used for the glucosinolates are slightly unorthodox when compared to those used by other papers (e.g. Brown et al. 2003). However, these codes are used consistently throughout the paper, including all figures, so they are retained in Table 1 so that readers can more easily locate the appropriate compound from codes given in the script.

Overall, given these problems with nomenclature, the findings reported concerning the effects of aphids on Arabidopsis glucosinolates still make sense in terms of known glucosinolate biosynthesis pathways. The aphidinduced decrease of two indole glucosinolates (3MIND and 4HIND) and increase of another (4MIND) would appear correct according to the biosynthesis pathway of 3 MIND $\rightarrow 4$ HIND $\rightarrow$ 4MIND (using our codes) given by Pfalz et al. (2016; see also Kim and Jander 2007). Foliage concentrations of 1MIND tended not to show strong or systematic changes when plants were challenged by aphids, which also makes sense as this compound is branched from this biosynthesis pathway (Pfalz et al. 2016). 
Table 1 Summary, classification and abbreviated codes of compounds extracted and measured from Arabidopsis foliage

\begin{tabular}{ll}
\hline Class and code & Compound \\
\hline Hormones & \\
JA & Jasmonic acid \\
ABA & Absicic acid \\
SA & Salicylic acid \\
SAGLY & Salicylate glycoside \\
Phytoalexin derivatives & \\
CAM & Camalexin \\
CAMA & Dihydro-camalexic acid \\
Amino acids & \\
TRP & Tryptophan \\
PHE & Phenylalanine \\
Flavonoids & \\
KRRG & Kaempferol-rhamnoside-rhamnoside- \\
& glycoside \\
KRR & Kaempferol-rhamnoside-rhamnoside \\
KGR & Kaempferol-glycoside-rhamnoside \\
Phenolic esters & \\
SM & Sinapoyl malate \\
FM & Feruloyl malate \\
Indolyl glucosinolates & \\
1MIND & 1-Methoxyindol-3-ylmethylglucosinolate \\
3MIND & Indol-3-ylmethylglucosinolate \\
4MIND & 4-Hydroxyindol-3-ylmethylglucosinolate \\
4HIND & 4-Methoxyindol-3-ylmethylglucosinolate \\
Aliphatic glucosinolates & \\
3MSUL & 3-(Methylsulphinyl)propyl glucosinolate \\
4MSUL & 4-(Methylsulphinyl)butyl glucosinolate \\
5MSUL & 5-(Methylsulphinyl)pentyl glucosinolate \\
6MSUL & 6-(Methylsulphinyl)hexyl glucosinolate \\
7MSUL & \\
8MSUL & \\
4MTHI & \\
5MTHI & \\
7MTHI & \\
\hline &
\end{tabular}

Acknowledgements The authors wish to thank Associate Professor Niels Agerbirk and Dr John Rossiter for insightful discussion and advice on these issues.

\section{References}

Brown PD, Tokuhisa JG, Reichelt M, Gershenzon J (2003) Variation of glucosinolate accumulation among different organs and developmental stages of Arabidopsis thaliana. Phytochemistry 62:471-481

Kim JH, Jander G (2007) Myzus persicae (green peach aphid) feeding on Arabidopsis induces the formation of a deterrent indole glucosinolate. Plant J 49:1008-1019

Pfalz M, Mukhaimar M, Perreau F, Kirk J, Hansen CIC, Olsen CE, Agerbirk N, Kroymann J (2016) Methyl transfer in glucosinolate biosynthesis mediated by indole glucosinolate O-methyltransferase 5. Plant Physiol 172:2190-2203

Publisher's Note Springer Nature remains neutral with regard to jurisdictional claims in published maps and institutional affiliations. 